RESEARCH ARTICLE

\title{
Inpatient Fall as Ominous Sign of Clinical Deterioration
}

\section{Fall as Ominous Sign}

\section{Irma Liyana Mushaddik ${ }^{1}$ MBBS, Karniza Khalid ${ }^{2}$ MBBS, MMedSc}

$1_{\text {Fall Prevention Team, Quality and }}$ Safety Unit, Hospital Tuanku Fauziah, Perlis, Ministry of Health Malaysia

${ }^{2}$ Clinical Research Centre, Hospital Tuanku Fauziah, Perlis, Ministry of Health Malaysia

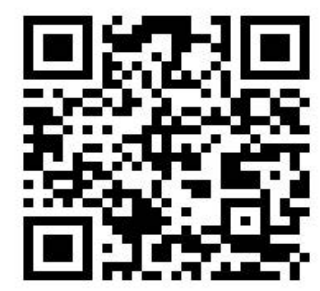

\begin{abstract}
:
Incidence of fall among hospitalised patients are commonly reported globally. Causes of inpatient falls are generally divided into extrinsic and intrinsic factors. Even though inpatient falls are commonly regarded as accidental with minor implications, we suggest that its incidence should be viewed with caution as it could be a red flag to clinical deterioration. Herewith we presented a clinical case scenario to delineate the importance to recognise fall as an early sign of clinical deterioration not otherwise detected from routine vital signs assessment.
\end{abstract}

Keywords: Falls, Vital Signs, Clinical Deterioration, Red Flag, Malaysia

\section{Introduction:}

Hospital falls are not uncommon and spur various prevention initiatives and reporting strategies worldwide [1-3]. It is generally associated with lengthy documentation and local investigation but only reported as part of the outcome statistics. Our tertiary hospital is the only governmental state healthcare institution in Perlis, Malaysia and our inpatient hospital falls were reported to be between 0.13 to $0.15 \%$ (Fig. 1).

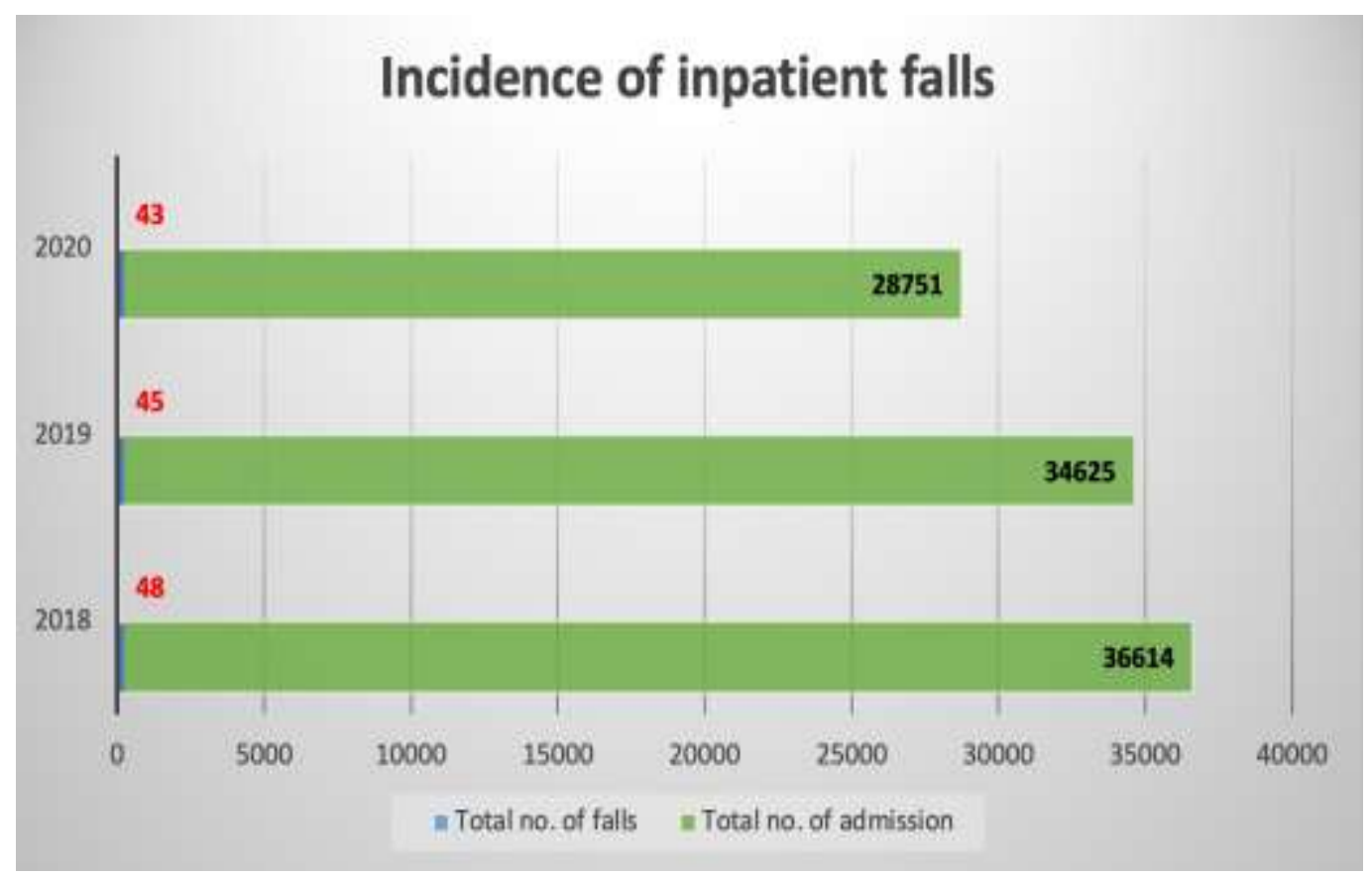

Fig. 1: Yearly incidence of inpatient hospital falls in Hospital Tuanku Fauziah, Perlis, Malaysia. Note: The numbers in black denote the total no. of admission while the numbers in red denote the no. of reported total inpatient falls. 


\section{Fall as Ominous Sign}

The outcome of inpatient fall varies from mild injury to death [4] which could be contributed by either intrinsic or extrinsic factors, or both. Intrinsic factors include factors related to the underlying disease dynamic including the severity of underlying infection, vestibular problem, movement disorders, and sensory deprivation, whereas extrinsic factors refer to external physical condition that may risk fall such as slippery floor, inappropriate footwear, tripping hazard or dim lighting from surrounding.

Fall is generally dismissed as mild and accidental event, particularly because a majority of the cases were minor and trivial [4]. However, we beg to differ and suggest that fall could be the first sign of imminent clinical deterioration prior to marked derangement in vital signs. Additionally, there is no standardised warning score system recommended for use in clinical practice to predict the risk of clinical deterioration hitherto, therefore early subtle changes in vital signs may be dismissed as normal variation.

We have encountered a few clinical cases of stable inpatients who reported trivial falls prior to abrupt clinical deterioration resulting in hospital death. However, we only learned in retrospect that these patients have had recent subtle decline in vital signs prior to the fall episode. One such example is a case of a 66-year-old male with underlying diabetes mellitus, hypertension, chronic kidney disease and ischaemic heart disease who presented with acute abdomen. He was treated for paralytic ileus due to electrolyte imbalance secondary to poor oral intake. His Morse Fall Scale (MFS) score was 45 on admission (presumed moderate risk for fall). He was otherwise clinically stable. However, he later developed hospital acquired pneumonia on day 10 of admission, requiring progressive escalation of oxygen therapy to face mask oxygen $5 \mathrm{~L} / \mathrm{min}$. Otherwise, he remained well and was weaned down to nasal prong oxygen within the following days. Regular routine monitoring of his vital signs were within normal limits and he was planned for completion of his intravenous course of antibiotics and further weaning of oxygen therapy.

However, a week later, the patient reportedly had one episode of fall by his bedside at around $7 \mathrm{pm}$.
The mechanism narrated was that he just got up from bed to urinate (in a urinal) by his bedside and fell on both knees when bending down. He denied prior dizziness, spinning sensation, or chest pain prior to fall. He sustained mild abrasion wound over his left forearm. Post-fall he was alert and well. His blood pressure post-fall was 107/69 $\mathrm{mmHg}$, GCS was 15/15, pulse rate $96 \mathrm{bpm}$, temperature $37^{\circ} \mathrm{C}, \mathrm{SpO}_{2} 99 \%$ on $\mathrm{NPO}_{2} 3 \mathrm{~L} / \mathrm{min}$ and dextrostix of $11.2 \mathrm{mmol} / \mathrm{L}$. However, in retrospect, it was noted that his baseline in-ward systolic blood pressure ranges from 130-150 $\mathrm{mmHg}$ and his baseline in-ward diastolic blood pressure ranges from 70-90 mmHg. That being the case, despite the stressful episode of fall which is expectedly to trigger some adrenaline rush and raises the blood pressure to some degree, is not observed in this patient. His routine blood panels were otherwise within normal limits and he did not require any special assistance following the fall episode. Unfortunately, during routine nursing observation round 4 hours later, he was noted to appear lethargic and had cold peripheries. His blood pressure was $87 / 46 \mathrm{mmHg}$ and he was gradually desaturating. Electrocardiogram (ECG) showed bradycardia with $\mathrm{T}$ inversion. Active resuscitation was initiated, however patient further deteriorated and passed away for acute coronary syndrome.

Therefore, such cases serve as a caution to the clinician whenever inpatient fall is reported. We routinely rely on the objective assessment of the vital signs during clinical monitoring, however when the changes were subtle, it is often dismissed as normal variation. Therefore, inpatient fall episode may be a red flag by itself as it may point towards a decline in haemodynamic stability. Failure to recognise fall event as a warning sign in the absence of marked disruption of vital signs may result in deleterious consequences on the patients.

\section{Corresponding Author:}

\section{Dr Irma Liyana Mushaddik}

Quality and Safety Unit, Hospital Tuanku Fauziah, Jalan Tun Abdul Razak, 01000 Kangar, Perlis, Malaysia

Email:irmaliyana@moh.gov.my 
Previous studies have looked at potential predictors to clinical deterioration in hospitalised patients and synonymously found that changes in vital signs may positively predict clinical deterioration [3,58]. However, subtle changes in blood pressure that would still be in normal range may be dismissed as part of daily, normal variation causing the sign to be ignored until the patient eventually collapsed. Inpatient fall events may result from various factors. However, in cases of hospital fall that is not attributable to extrinsic factors, the most plausible explanation would possibly relate to haemodynamic instability causing labile and fluctuating blood pressure, resulting in temporary altered mental status or physical weakness leading to fall. Therefore, the percipience of the HagenPoiseuille law would be relevant when studying the potential factors involved in maintaining blood pressure in vivo. The law stated that the pressure difference between two points is a function of resistance and blood flow [9]. Resistance on the other hand, is directly proportional to the blood viscosity and inversely proportional to the fourth power of the arteriolar radius. Therefore, it is not surprising that the arterial blood pressure is perhaps the most finely regulated cardiovascular variable in the human body, and is accomplished by regulating the radii of the vessels (arterioles and metarterioles) within the organ system.

It is important to note that a small change in vessel radius will have an inverse effect on its resistance to flow by the fourth power. In other words, an increase in vessel diameter by $50 \%$ will decrease its resistance to flow by approximately 16 -fold, resulting in a drop in blood pressure. Therefore, in the setting of septicaemia, which is one of the common causes of hospital admission [10], robust immune response with recruitment of inflammatory mediators would be anticipated, resulting in widespread vasodilation, hence lowered blood pressure. This response is exaggerated in the setting of defective intrinsic autoregulation mechanism, which a common observation in progressive disease dynamics. Therefore, these patients may have a comparatively lower blood pressure as compared to their baseline, leading to fall especially when they are changing postures. Hence, the development of an early warning score system to identify patients at risk of clinical deterioration should also include fall as a significant variable in the prediction model.

We would suggest using a detailed post-fall clerking sheet to assist the clinicians to identify early warning signs of clinical deterioration. There should be a mandatory assessment of postural blood pressure as recommended in the Fall Guidelines Ministry of Health Malaysia 2019 as postural drop is an early sign of haemodynamic instability. Additionally, the identified intrinsic and extrinsic factors for the risk of fall may help the clinicians to be more vigilant by performing a more thorough assessment and plan appropriate remedial actions.

\section{Conclusion:}

Events of inpatient fall are not uncommon in the clinical setting. The occurrences are naturally dismissed as accidental due to the commonly associated trivial mechanism. Fall is multifactorial, but could be a tell-tale sign of haemodynamic instability as subtle changes in vital signs may be missed during routine monitoring. Therefore, we aim to highlight the importance to recognise inpatient fall events as warning sign to clinical deterioration and such incidence should trigger caution and intensive care.

\section{Acknowledgement:}

We would like to thank the Director General of Health Malaysia for his permission to publish this article.

\section{References:}

1. Jørgensen TSH, Hansen AH, Sahlberg M, Gislason GH, Torp-Pedersen C, Andersson $\mathrm{C}$, et al. Nationwide time trends and risk factors for in-hospital falls-related major injuries. Int J Clin Pract. 2015; 69(6):7039.

doi: http://doi.wiley.com/10.1111/ijcp.12624

2. Morgan VR, Mathison JH, Rice JC, Clemmer DI. Hospital falls: A persistent problem. Am J Public Health. 1985; 75(7):775-7. 


\section{Fall as Ominous Sign}

doi: http://ajph.aphapublications.org/

3. Hendrich A, Nyhuis A, Kippenbrock T, Soja ME. Hospital falls: development of a predictive model for clinical practice. Appl Nurs Res. 1995; 8(3):129-39.

4. Hitcho EB, Krauss MJ, Birge S, Dunagan WC, Fischer I, Johnson S, et al. Characteristics and circumstances of falls in a hospital setting. J Gen Intern Med. 2004; 19(7):732-9.

doi:http://link.springer.com/10.1111/j.15251497.2004.30387.x

5. Churpek MM, Yuen TC, Edelson DP. Predicting clinical deterioration in the hospital: The impact of outcome selection. Resuscitation. 2013; 84(5):564-8.

6. López-Soto PJ, Smolensky MH, SackettLundeen LL, De Giorgi A, RodríguezBorrego MA, Manfredini R, et al. Temporal Patterns of In-Hospital Falls of Elderly Patients. Nurs Res. 2016; 65(6):435-45. doi:http://journals.lww.com/00006199-
201611000-00003

7. Brekke IJ, Puntervoll LH, Pedersen PB, Kellett J, Brabrand M. The value of vital sign trends in predicting and monitoring clinical deterioration: A systematic review. Patman S, editor. PLoS One. 2019; 14(1):e0210875.

doi: https://dx.plos.org/10.1371/journal.pone.0210875

8. Churpek MM, Adhikari R, Edelson DP. The value of vital sign trends for detecting clinical deterioration on the wards. Resuscitation. 2016 M;102:1-5.

9. Vanderpool RR, Naeije R. Hematocritcorrected pulmonary vascular resistance. Am J Respir Crit Care Med. 2018;198(3):305-9.

10. Chang DW, Tseng $\mathrm{CH}$, Shapiro MF. Rehospitalizations following sepsis: Common and costly. Crit Care Med. 2015;43(10):2085-93.

How to cite this article: Mushaddik, I. L., \& Khalid, K. (2021). Inpatient Fall as Ominous Sign of Clinical Deterioration. Journal of Current Medical Research and Opinion, 4(02). https://doi.org/10.15520/jcmro.v4i02.395 